\title{
Design Patterns for Mixed-Method Research in HCl
}

\author{
Koen van Turnhout, Arthur Bennis, Sabine Craenmehr, Robert Holwerda, Marjolein Jacobs, \\ Ralph Niels, Lambert Zaad, Stijn Hoppenbrouwers, Dick Lenior, René Bakker. \\ HAN University of Applied Sciences \\ Academy of Information Technology and Communication \\ [Koen.vanTurnhout, Arthur.Bennis, Sabine.Craenmehr, Robert.Holwerda, Marjolein.Jacobs, \\ Ralph.Niels, Lambert.Zaad, Stijn.Hoppenbrouwers, Dick.Lenior, Rene.Bakker] @ han.nl
}

\begin{abstract}
In this paper we discuss mixed-method research in HCI. We report on an empirical literature study of the NordiCHI 2012 proceedings which aimed to uncover and describe common mixed-method approaches, and to identify good practices for mixed-methods research in HCI. We present our results as mixed-method research design patterns, which can be used to design, discuss and evaluate mixedmethod research. Three dominant patterns are identified and fully described and three additional pattern candidates are proposed. With our pattern descriptions we aim to lay a foundation for a more thoughtful application of, and a stronger discourse about, mixed-method approaches in HCI.
\end{abstract}

\section{Author Keywords}

Mixed-method research; methodology; triangulation.

\section{ACM Classification Keywords}

H.5.m. Information interfaces and presentation (e.g., HCI): Miscellaneous.

\section{INTRODUCTION}

The work presented in this paper is part of a long-term research effort which addresses mixed-method research in the context of multidisciplinary HCI research and of research education for HCI professionals. Mixed-method research is common in HCI [37], but there is little literature to support the design of mixed-method studies in our field. Also, we notice authors do not typically refer to their research as mixed-method research. Specifically, they tend not to make explicit how the components of their research, often borrowed from several contributing disciplines, fit together - ideally in such a way that 'the whole is more than the sum of its parts' [3,7]. We do think HCI researchers make sound pragmatic decisions when applying

\footnotetext{
Permission to make digital or hard copies of part or all of this work for personal or classroom use is granted without fee provided that copies are not made or distributed for profit or commercial advantage and that copies bear this notice and the full citation on the first page. Copyrights for thirdparty components of this work must be honored. For all other uses, contact the Owner/Author.
}

Copyright is held by the owner/author(s).

NordiCHI'14, Oct 26-30 2014, Helsinki, Finland

ACM 978-1-4503-2542-4/14/10.

http://dx.doi.org/10.1145/2639189.2639220 a mixed-method approach, but their practice of mixing methods does not seem to be matched by explicit underlying considerations. We believe that closing this practice-theory gap would help to better teach, discuss, design and evaluate the mixed-method research approaches which our community uses.

A possible cornerstone of a theory for mixed-method research design is the Development Oriented Triangulation (DOT) framework [37], which offers a classification of methods organized around trade-offs researchers need to make in their research planning. We used this framework in an empirical literature study, which is a common approach for addressing methodological questions like the ones addressed in this paper - see for example [7,29,36]. Through an in-depth analysis of a large portion of the NordiCHI 2012 proceedings, we identified common method mixes and the types of problem they addressed. We identified best practices for each mix through a comparison and critical discussion of papers adopting a similar mix.

We have chosen to represent our findings as mixed-method research design patterns. Originated by Christopher Alexander [1], design pattern languages have become a popular way to represent middle level design knowledge [32], which is used for software architecture [21] and interaction design [3], among other fields. Though we adopted the format of design patterns for mixed-method research designs, the results of our study can only provide a first step towards a full-fledged pattern language.

This paper is organized as follows. We first discuss existing work about mixed-method approaches within HCI, Information Systems and the Social Sciences. Next, we provide an in-depth discussion of the DOT-framework and some of its foundations. We then turn to the setup and results of the empirical literature study and we discuss the common patterns we found. We finish the paper with conclusions and a discussion of the work done.

\section{RELATED WORK}

Many authors place the field of HCI at the crossroads of several branches of science, engineering and design $[1,18$, 20, 26, 34, 39]. Historically, science and engineering may have been the most dominant cultures in HCI [1,18,29,38], but recent years a successful emancipatory movement has made a case for design and design research as a means to 
produce relevant knowledge [13,14,24,25,40]. This revitalized influx of design-oriented work has been crucial to third wave HCI [4] and it has stipulated once more that design, in its many incarnations, is widely recognized as a core means of knowledge production in HCI [1,6,14, $21,25,34,39,40]$.

In response to the linguistic confusion and tensions between the several contributing disciplines in HCI [14], several authors have argued for a cross-disciplinary methodology in HCI $[26,28,33]$. Inspiration for such a methodology can be drawn from the social sciences, where mixed-method approaches have shown to be capable of overcoming the ontological (objects of study), epistemological (approaches for knowledge production) and axiological (values in knowledge production) differences which were fiercely debated during the 'period of the paradigm wars' $[3,12]$. Currently, mixed-method designs with solid knowledgetheoretical underpinnings do exist for the social sciences $[12,16]$.

However, while many of the results in social science literature, such as common reasons for mixing methods [7, 16], may be appropriate for HCI, the foundations of this work have to be reconsidered thoroughly. A core difference between social sciences and HCI, for example, is the status of theory. Being a design-oriented field, HCI strives to combine descriptive and prescriptive theory [17,22] and recognizes (annotated) artefacts as a legitimate form of knowledge [8,15,17,22,25,29,34,39,40].

This has consequences for the knowledge production practices and the way we cluster them. It is hard to imagine how thinking of a mixed-method design as a combination of qualitative and quantitative methods -as it is defined in most social science textbooks- can relieve the common tension between understanding oriented work and creation oriented work [6,14] (to name but one example). To advance mixed-method approaches for HCI, mixed-method theory has to be developed, including an ontology, epistemology and axiology fitting our field. The DOTframework, to which we turn next, is an effort to do just that.

\section{DEVELOPMENT ORIENTED TRIANGULATION}

Overview of the DOT-framework

Figure 1 shows the DOT-framework. It identifies research strategies, which are organized along 3 central trade-offs which HCI researchers face when choosing one method over the other.

\section{First layer: Two domains for $\mathrm{HCl}$ Research}

In the ontological top layer, the DOT-framework follows Hevner [22] and Mackay and Fayard [26] in identifying two domains of study for HCI-professionals [37]. Both domains are a resource for researches as well as an opportunity space for change. The first domain is the application domain: HCI researchers need to learn how humans interact with computers and they aim to change this interaction for the better. The second domain is the domain of available work. This consists of existing artefacts, theories and models which a researcher can have access to. HCI researchers study available work and contribute to it. All HCI research activities take place in the innovation space between these two domains. The DOT framework thus casts HCI research as an "organized learning activity which is instrumental to an innovation or development challenge and, as such, brokers between the domain of available work and the application context" [37].

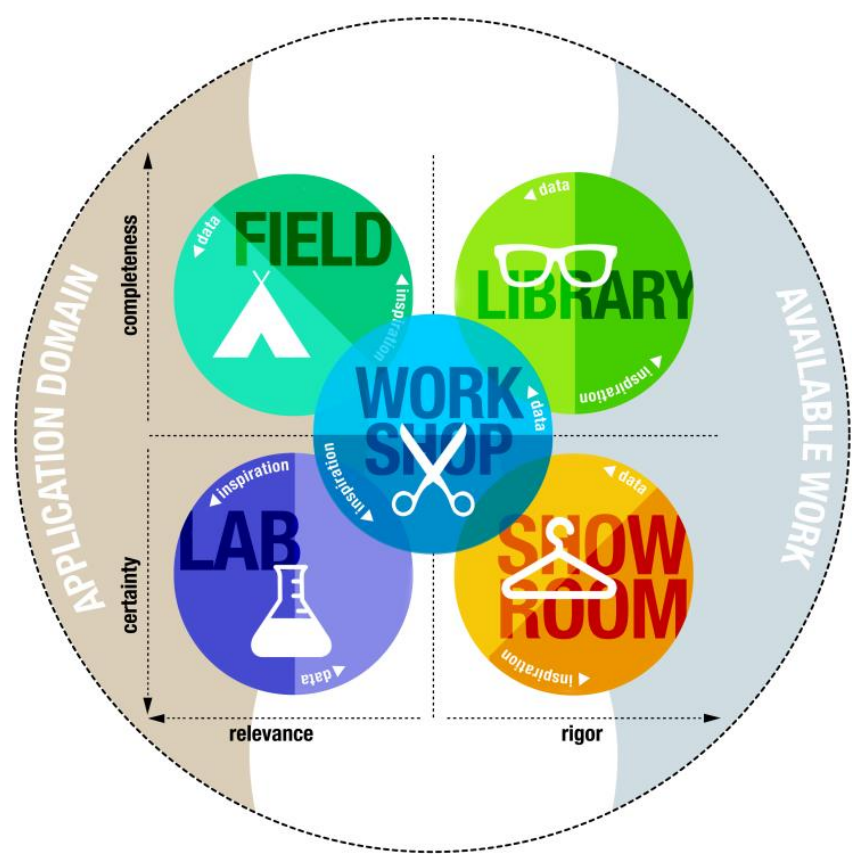

Figure 1: Overview of the DOT-framework showing five types of research, related to two domains and three fundamental trade-offs.

\section{Second layer: Three trade-offs}

The second layer of the DOT-framework is axiological in nature. It identifies three trade-offs between basic values in research design which cannot be optimized simultaneously and thus need to be triangulated [26,37].

\section{Rigor or Relevance?}

The distinction between the two domains in the top layer directly translates into a research trade-off. Hevner et al. [22] propose that there are multiple 'research cycles'. The researcher learns about and changes the application domain in the relevance research cycle. In the rigor research cycle, in contrast, researchers learn about and contribute to available work. Thus defined, rigor and relevance need to be triangulated: they are both long-term goals for $\mathrm{HCI}$ research, but they are hard to optimize simultaneously within a single research strategy.

Certainty or Completeness?

Second, the framework identifies the trade-off between certainty and completeness. This is taken from [35] who distinguish between the concerns for 'precision of measurement' and 'system character of context'. According 
to [35], researchers choosing precision of measurement would need to use laboratory experiments or judgment tasks while researchers who value the system character of context would use field studies or ethnography instead.

\section{Inspiration or Data?}

The third trade-off in the DOT-framework is between those approaches that require researcher involvement and subjectivity (called inspiration-oriented approaches in the framework) and those that view the researcher as independent observer of reality (called data-oriented approaches). Inspiration-oriented approaches have also been labeled 'intuitive' [14], 'phenomenological' [13, 20] or 'creative' design [39]. Data-oriented approaches are also known as 'analytic' [14], 'positivistic' [13] or 'engineering' design [39].

\section{Third Layer: Five research strategies}

Third, in the epistemological layer, the DOT-framework replaces the distinction between qualitative and quantitative methods as used in the social sciences by five distinct research strategies for HCI. These are aligned with the trade-offs in the second layer of the framework, leading to a close mapping of epistemology and axiology - also found in [35]. This connection between the second and third layer sets the classification of methods in the DOT-framework apart from other classifications of methods (see [25] for an overview), which lack such an underpinning. Nevertheless, the final classification is close to that of [30].

\section{Library}

Library methods enable researchers to learn about available work related to their research question. Literature studies, the creation of a benchmark and competition analyses are typical library studies. Library studies may be inspirational or data oriented, and they aim to get a better connection with and an overview (completeness) of available work (rigor) which is relevant to the research problem.

\section{Field}

Field methods, often borrowed from interpretive social science [31], aim to capture the context of design [25], or in the terms of the DOT-framework: to get a complete understanding (completeness) of the application domain (relevance) of the development effort. Some field methods, such as contextual inquiry, show a strong reliance on data gathering and analysis while others such as cultural probes are explicitly optimized to be inspirational for the designers involved. Field methods are found in [30] as 'observation', and in [25] as 'field methods'.

\section{Workshop}

Within the DOT-framework workshop methods are defined as methods which aim to conceive or improve the solution without a direct reference to the domain of available work or application context. Most software engineering disciplines [22,31] and the 'research through design' community $[25,40]$ consider creating artifacts an important part of research and development efforts. The creative design [39] tradition has developed many inspiration- oriented methods such as ideation methods and morphological maps, while engineering design [39] typically relies on more analytical workshop methods such as optimization metrics, iterative improvement of the system performance, or code refactoring. Workshop methods are more narrowly defined in [30] as 'systems development'.

\section{Lab}

Lab studies aim to test (certainty) a proposed solution, against aspects of (or goals for) the application domain (relevance). They typically involve some form of empirical manipulation, if only as lightweight as asking users to try a prototype of a new system. Lab studies complement field studies with their concern for the application domain, but field studies are more suitable for getting an overview, while the lab studies aim at optimizing the certainty of the outcomes through controlled experimentation. Most usability evaluations are lab studies. In [25] the authors use the term 'lab study' in the same fashion as we do, while [30] calls it 'experimentation'.

\section{Showroom}

The DOT-framework identifies showroom methods as methods that help to make specific work (certainty) more reusable by other researchers (rigor). One example is the explicit comparison of the performance of an algorithm, with a benchmark. Another example is a critique of a (finished) design in relation to existing work; as this helps others to assess the potential of the proposed solution for their problem. The creation of design frameworks or guidelines intended to highlight considerations that go beyond individual designs are also labeled 'showroom methods'. In [30] the combination of library and showroom studies is called 'theory building'; in [25] the term 'showroom' is used, be it in a somewhat narrower sense.

\section{AN EMPIRICAL LITERATURE STUDY}

\section{Setup}

The basis for the work presented in this paper is a classification of an a-select sample of the NordiCHI 2012 proceedings using the DOT-framework. Scholarly papers are often used as a shortcut to understand scholarly practice (e.g. [7,29,36]). Although academic papers may represent a slightly stylized version of the actual work, we had enough confidence in the transparency realized by the authors to assume that all relevant details that we needed for defining patterns could be found in the papers. We chose NordiCHI because it is one of the larger European conferences on $\mathrm{HCI}$, thus providing a fair selection of the HCI research in Europe in proceedings of manageable proportions (100 papers in total). It was also the most recent European HCI conference when we first started this study. The study was carried out by a group of 7 raters. All raters were staff members of our university involved in research, teaching, or both. In an earlier study [37] a smaller sample of 10 papers of the same proceedings were examined as a first test of the applicability of the DOT-framework for this 
purpose. Only one rater of the current group was also involved in that study.

The first phase of the study comprised of a reexamination of the ten papers which were studied in [37]. This allowed us to critically reexamine the reported triangulation paths of this study, to get acquainted with the task of classifying papers with the DOT-framework and to sharpen our procedures. In this phase at least four raters read each paper and extensively discussed their interpretations to reach consensus on the triangulation paths reported in the paper. The resulting consensus differed from the interpretation in [37] for two papers, and only on minor points. Nevertheless, substantial differences in the 'first reading' of the papers existed, and we found it necessary to adjust procedures followed in [37] to increase the ease of replication.

As in [37], we considered the narratives in the papers as a report of a triangulation path: a string of smaller and bigger chunks of research which can be labeled with the research strategies of the DOT-framework and which typically 'crosses' one or more of the trade-offs of the framework. Reconstructing this path from a paper is not trivial. Paper narratives transcend several layers of abstraction. For example: a paper may have the goal to deliver a novel theory (showroom) about HCI practitioners in the field (Figure 2, top layer), which is then realized at a lower level of abstraction by combining a field, library and showroom study (Figure 2, middle layer). On a yet lower level of abstraction (Figure 2, bottom layer) this may involve activities which could be described as workshop and lab activities.

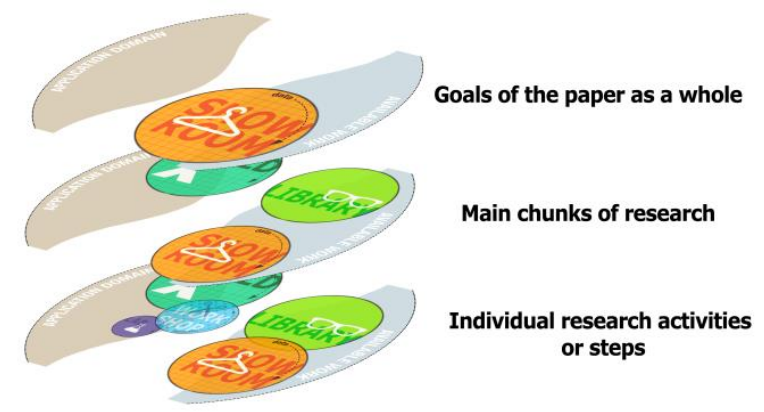

Figure 2: Papers can be read at different levels of abstraction, each rendering its own 'reading' of the triangulation path. In practice the layered picture drawn here is an idealization, too.

To arrive at a uniform classification of the triangulation path, we need to reconstruct these layers in the exegesis of the paper. This is complicated by the fact that not all research activities are equal in size and (seemingly) in importance to the authors. As [37] report, the separate chunks in a research paper may differ in size and may be reported incompletely (e.g. reporting results only, rather than the complete research cycle). In [37] these problems were addressed by making a distinction between the thick path (main chunks of research) and thin path (concerns that were addressed in a lightweight manner) of the paper. This distinction, however, turned out to be sensitive to disciplinary bias of the raters and the decision whether a chunk should be considered 'not present', 'thick', or 'thin' was a source of debate. Therefore a sharpened procedure and more objective criteria for the 'weight' of a chunk were put in place.

To address the layered nature of papers we made a prediction of the triangulation path based on the abstract and introduction of the paper, which then was verified with a close reading of the full paper. This procedure sensitized raters for the hierarchies as they were constructed by the authors of the papers (the principle of 'authors' intent'). Also we agreed not to divide a fully reported research cycle into multiple chunks. For example, if authors gathered field data and described how they processed it to arrive at conclusions, this was considered as part of the field study and not as new chunk of research in need of classification. Also, we replaced the 'thin' and 'thick' distinction with a set of four, less ambiguous, criteria: the SPIM criteria (Table 1).

Table 1: The SPIM criteria for deciding on the 'weight' of a chunk of research.

\begin{tabular}{ll}
\hline $\mathrm{S}$ & Separate Contribution \\
$\mathrm{P}$ & Paper Space \\
$\mathrm{I}$ & Internal Impact \\
$\mathrm{M}$ & Method Transparency \\
\hline
\end{tabular}

With separate contribution (S) we refer to the idea that a chunk of research can be seen by the authors as an independent contribution to the field of HCI, for example if they mention it as such in the abstract or introduction. With paper space $(\mathrm{P})$ we refer to authors showing they find a part of their work important by dedicating paper space to it (the criterion was scored when authors dedicated more than $1 / 5$ th of the paper to the research chunk). Internal impact (I) refers to the idea that ideally, in a mixed-method paper, each chunk of research has an influence on other chunks. Internal Impact was scored when it was possible to infer how a chunk impacted the rest of the study. The final criterion was method transparency $(\mathrm{M})$ which we used to indicate whether the authors were transparent about the methods they used to answer the questions of their study. Together the SPIM scores gave us a much more objective and nuanced view on how authors dealt with a chunk of research than in the earlier study, i.e. [37].

Another difficulty with the classification of papers is dealing with chunks that show characteristics of multiple research strategies. For example, co-design workshops typically focus on creating new solution directions. Stakeholders from the application domain are often present both to give 'field' input, and to 'validate' early solutions. In participatory design settings, these workshops are often executed on site. Thus, co-design workshops share 
characteristics with both field studies and lab studies. Similar 'confusions' arose between lab and field (when an intervention was lightweight, for example) and showroom and workshop (some raters scored the 'construction' of a framework as workshop, while others preferred showroom). We documented the disagreements and consensus decisions and this documentation was consulted by the other raters in case of doubt.

Within the revised setup we then set out to classify a set of 30 more papers from the NordiCHI proceedings. Each paper was rated by two raters from our group, in new pairs for each paper. The two raters classified the paper independently reached consensus on the triangulation path. The consensual outcome and possible remaining discussion points were discussed in the whole group. All raters had read and classified the abstract and introduction, giving them enough background to act as a sparring partner for the raters who had read the full paper. Cohen's kappa was calculated for all independent ratings (before the consensus meeting). Kappa was calculated by treating all 25 decisions a rater had to make: the four SPIM decisions for all research strategies, as well as the decision whether a study should be regarded as inspiration or data oriented - as independent nominal decisions. This gave a somewhat conservative estimate of agreement, but we found it more suitable than, for instance, combining the SPIM criteria in an ordinal agreement measure. This procedure led to an estimated value of Kappa (Fleiss Kappa, with Conger's correction) of $\hat{\kappa}_{C}=0,70$ which can be considered good [19]. Consensus was also easily reached in most cases suggesting that remaining differences in first judgment were small.

\section{Results}

To give an overall idea of the classifications, Table 2 lists the number of times a research strategy was identified in a paper, and whether it was scored as an inspiration or data oriented method. The table shows all research strategies were found frequently in our dataset and a fair division between inspiration and data oriented approaches was found. It appears that the three dimensions of the framework give a balanced coverage of research approaches in HCI.

Table 2: totals for scored research strategies in the dataset.

\begin{tabular}{c|c|c|c|} 
& Inspiration & Data & Total \\
\hline Library & 29 & 9 & 38 \\
\hline Field & 6 & 10 & 16 \\
\hline Workshop & 16 & 8 & 24 \\
\hline Lab & 11 & 15 & 26 \\
\hline Showroom & 14 & 10 & 18 \\
\hline
\end{tabular}

Not all research strategies scored equal on all SPIM criteria. Table 3 shows separate counts for those (note that multiple SPIM values are common for each scoring of a research strategy).
Table 3: SPIM criteria for research strategies

\begin{tabular}{l|r|r|r|r|} 
& $\begin{array}{l}\text { Separate } \\
\text { contribution }\end{array}$ & $\begin{array}{l}\text { Paper } \\
\text { Space }\end{array}$ & $\begin{array}{l}\text { Internal } \\
\text { impact }\end{array}$ & $\begin{array}{l}\text { Method } \\
\text { transparency }\end{array}$ \\
\hline Library & 14 & 9 & 37 & 6 \\
\hline Field & 11 & 8 & 15 & 14 \\
\hline Workshop & 22 & 16 & 22 & 14 \\
\hline Lab & 20 & 16 & 16 & 9 \\
\hline Showroom & 23 & 14 & 14 & 18 \\
\hline
\end{tabular}

It is shown that, while field, workshop and lab studies have a good distribution across the SPIM criteria, library studies and showrooms do not. Showroom and library score low on method transparency, indicating that authors typically mention results of these studies (such as reviews of existing literature, novel guidelines or frameworks), but not how they arrived at their results. The low frequency for the I criterion for showroom is explained by the fact that showroom is typically found in the last section of the paper, for which we did not score this criterion.

\section{TOWARDS RESEARCH DESIGN PATTERNS}

\section{Approach}

Having decided on the full triangulation paths of all papers, we clustered the papers which had a similar triangulation path. We also looked at the contents of the papers, using the contribution types in HCI as discussed by Newman [29], Cockton [10] and the CHI 2013 organization [9]. This led to a clustering of papers within pattern proposals. We placed papers which were arguably similar in, eventually, six pattern proposals. It turned out that three quarters of the papers in the set were covered by only three dominant patterns. Only two papers could not be classified in any of the six pattern proposals. Table 4 lists the division of papers across (candidate) patterns.

\section{Table 4: Patterns which were found}

Pattern name

NO papers

\begin{tabular}{|l|l|}
\hline Rigor Cycle & 9 \\
\hline Validated Solution & 11 \\
\hline Field Reframing & 10 \\
\hline Parameter Discovery & 4 \\
\hline Transformative Design & 3 \\
\hline Relevance Cycle & 1 \\
\hline Unclassified & 2 \\
\hline
\end{tabular}

To make the step from clusters of papers to patterns -which are partially prescriptive - the sets of papers belonging to a pattern proposal were revisited and discussed thoroughly in the group of 7 raters. For our pattern descriptions it was important to decide why strategies were combined in the papers that we studied. Therefore we maintained a list of possible combination goals. We revised the list several 
times, striving to balance comprehensiveness, precision and parsimony and by comparing it with the lists offered for the social sciences by $[7,16]$. Table 5 shows our final set of combination goals. Although we could fit the reasons for combining methods to our set, we do not claim the list is complete for all HCI studies in general.

Table 5: Reasons for combing methods in HCI

\section{Shorthand: Description}

Niche: One study delineates or identifies a space which can be filled in a later study. A niche can be explored, filled, or illustrated (with a concrete example of a general idea).

Proposition: A study delivers a result, insight or prototype which can be tested or expanded in a follow-up study. A proposition can be tested, validated, positioned or expanded (placed in a broader context)

Framing: A study delivers context, a corresponding background understanding, a more or less coherent way of thinking about a problem. A frame can be illustrated, transformed, or expanded.

Content: A study is done to collect concrete materials such as a dataset or test-setup which can be used in a follow up study. Content can be analyzed, or used.

Guidance: One study delivers insights which help to set up a follow up study. Guidance can be followed.

Finally, we compared the papers in each pattern to the standards that can be derived from the DOT-framework. In particular we looked at whether triangulation across one or more of the trade-offs in the framework occurred within patterns and we considered to what extent the patterns could be combined with other, complementary, patterns. Moreover, we consulted external standards for best practice research as given by the $\mathrm{CHI}$ organization [9] and others $[17,22,25,29]$. Matching the existing principles and standards with our experiences in reading the papers resulted in pragmatic research standards which would fit each pattern. In the next section we discuss the dominant patterns which were the result of this effort.

\section{THREE DOMINANT PATTERNS}

\section{Validated Solution}

\section{Use when}

We define validated solutions as studies which propose new artefacts, infrastructures or interaction techniques. Starting point for this approach can be an unsolved problem, deficiencies in existing solutions, or the need to illustrate a novel vision or idea with concrete examples.

\section{Why}

The validated solution is an effective way to further the state of the art and to 'push' new ideas and interaction techniques. It can efficiently bridge rigor and relevance, although some lab studies fail to touch the 'real' application domain if this is not clearly defined upfront.

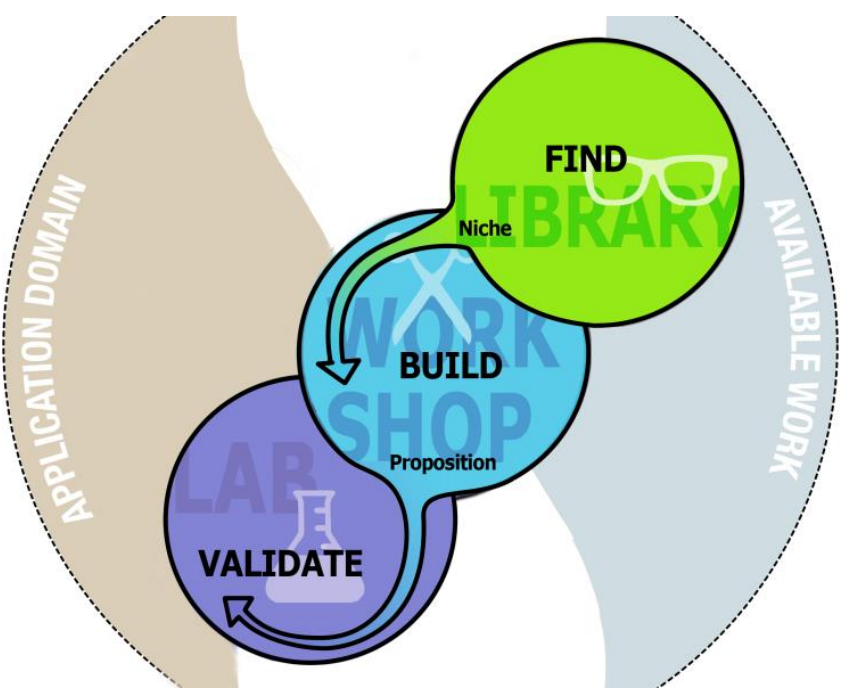

Figure 3: Triangulation path for a validated solution paper.

How

Figure 3 shows the typical triangulation path for a validated solution. A library study can be used to provide context about related solutions and to set a scope (identify a niche) for the rest of the study. In a workshop study, a solution is developed as an illustration of the ideas which have been the starting point for the work. The solution then acts as a proposition which is tested with users in a lab study. In [f], for example, the feasibility of location based voice messaging (identified niche) is demonstrated by building a prototype (proposition) and testing it on technical reliability and acceptability for users (validation).

For this triangulation path it is important that the authors provide argumentation about the perceived advantages of the intended solutions - usually in response to deficiencies of existing solutions [9,29]. The solution needs to be described in sufficient detail, so others can replicate it $[9,17]$. The validation needs to be rigorous [9], which means it is ideally data oriented. For effective triangulation of completeness and certainty it is important that the solution is evaluated against the perceived advantages which were outlined at the beginning and arise from existing work. Proper triangulation of rigor and relevance suggests the lab study should mimic the intended context of use as closely as possible. If the work is more explorative in nature, these standards may be applied less rigorously, but we advise to add a showroom study to prepare a follow-up by other researchers.

\section{Special Cases and Combinations}

A showroom study is sometimes added to the validated solution and we recommend this in particular for more explorative papers. The lab study can be split into multiple lab studies, of which some focus more on the evaluation of robustness of the system and others on the subjective experience of users. The pattern can be combined with the 
rigor cycle and act as a good follow-up on the field reframing pattern.

\section{Paradigm Papers}

The validated solution pattern was found in 11 of the 40 papers. The aforementioned study [f] uses multiple lab studies. In Dalsgaard et. al. [b], a validated solution approach for 3D tangible tabletops is combined with a showroom study aimed at explicating design considerations.

\section{Rigor Cycle}

\section{Use when}

The rigor cycle can be used to explore solutions and to weed out problems in existing work. Within our dataset we found the rigor cycle approach for two types of papers. First we found it for improved methods in which deficiencies in existing methods are identified, solved and evaluated against the literature. Second, we found it in papers that are in the early stages of exploring novel ideas.

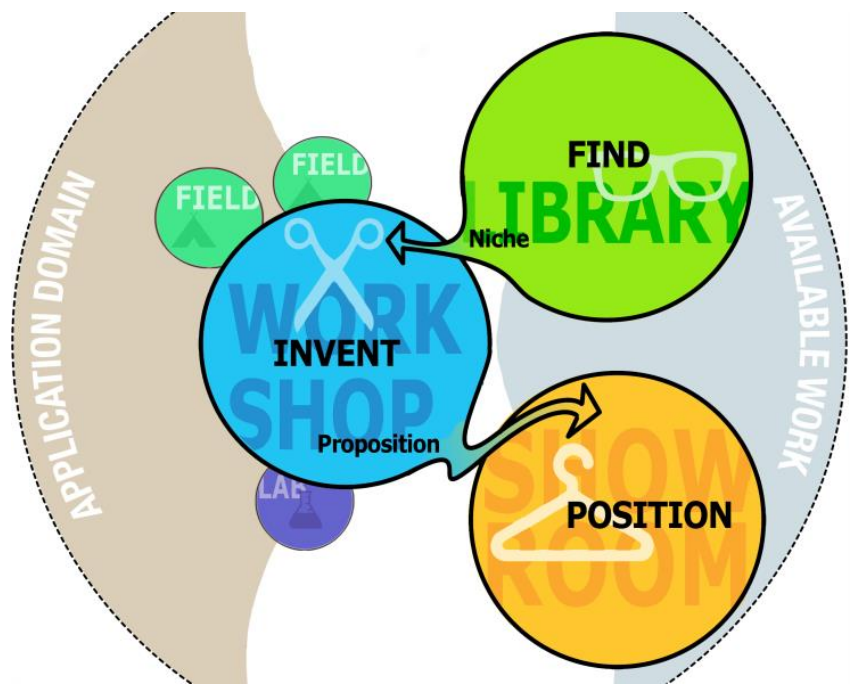

Figure 4: Triangulation path for a rigor cycle paper in HCI.

\section{Why}

The rigor cycle approach relates new work explicitly to existing work, enabling a long term development of the state of the art. Both types of rigor cycle papers as found in our set appeared to provide a sensible alternative for the validated solution approach. User validation of improved methods is cumbersome and when clear requirements or arguments for a new method can be formulated based on existing work, a user validation may not always be necessary. In explorative design studies, understanding the proposed solutions from the point of view of available work may be more urgent than validating these (immature) solutions with users.

\section{How}

Figure 4 shows the triangulation path for the rigor cycle, including the reasons for mixing methods. Typically a library study identifies a niche which is illustrated with the solution as invented in the workshop. Workshop studies in this pattern can involve users in some form, i.e. through an embedded field study, or by involving stakeholders in codesign activities. The result of the workshop study is a proposition which can be positioned against available work, for example by showing how existing deficiencies are solved in the solution.

Triangulation of completeness and certainty can be achieved by identifying requirements for the solution (or method) and evaluate the result explicitly against those requirements. The design of the solution ought to be described in a replicable (thus data oriented) way $[9,17]$. Triangulation of rigor and relevance is at risk in this pattern, so user involvement in the workshop study is necessary. This can be done by involving users in a co-creation session or by using a real world dataset as content in the workshop. Exploratory research efforts can take more liberty in the way several activities within the pattern are executed, and for those an inspiration oriented approaches may even be preferable. If so, the methods need to be clearly described and a well-executed showroom study is vital for an effective contribution to the field.

\section{Special Cases and Combinations}

A field or lab study could be added to make sure the work has a better fit with the application domain. Papers oriented at improving methods can use a field study as content for the workshop. The validated solution pattern forms a natural complement to the rigor cycle pattern.

\section{Paradigm Papers}

The rigor cycle was found in 9 of the 40 papers. A good example of a rigor cycle approach to improving methods is found in [g], as it shows how correspondence analysis can improve certain aspects of the persona segmentation process. A particularly interesting paper of the exploratory kind is [e] who applied the pattern several times to arrive at guidelines for technology enhanced dance performances.

\section{Field Reframing}

Use when

Field reframing can be used when a particular context of use is of interest but not yet studied from a particular point of view. In our dataset we found it was used (1) to understand HCI professionals in the field, (2) to understand users working with emergent technologies (such as novel uses of smartphones) and (3) as a starting point for the design of novel interfaces. We found understanding users and theory to be the most common categories [9] for field reframing papers.

Why

The field reframing pattern can deliver generic findings which are useful for many design problems. The pattern can also bring in the 'real' world, or 'user pull' which is lacking in validated solutions and rigor cycles.

How

Field reframing papers typically use a library study to understand kernel theories about a problem area, and to identify a niche for the field study, or to frame it. This 
frame is reconsidered in the field study where novel insights about users are gathered. This, in turn, gives guidance and framing to the showroom study, which transforms the findings in a reusable format and gives it new meaning in relation to existing work. Showroom studies in this pattern show a synthesis of findings from the field and from the literature (Figure 5). Brown et al. [a], for example, used activity theory as a theoretical framework from which they studied how interaction designers and developers in the field aligned their work implicitly. They position their results in relation to existing theories.

The library study needs to be aimed at a deep understanding as it needs to both frame the field study and feed the synthesis in the showroom study at the end. While the field study is typically the main focus of the work (and as such it needs to be executed in a rigorous way [9]), we found that the execution of the showroom study also had a large impact the quality of the field-reframing papers. As the showroom study is preparing the follow up, it supports the combinability of this pattern with others. The showroom study could relate the novel findings to existing work for long term theory development with a post-study literature review, as is done in [a], or transform the insights gained in the field into guidelines or a framework which is directly applicable by designers. Typically the showroom is where the step from descriptive to prescriptive theory is made.

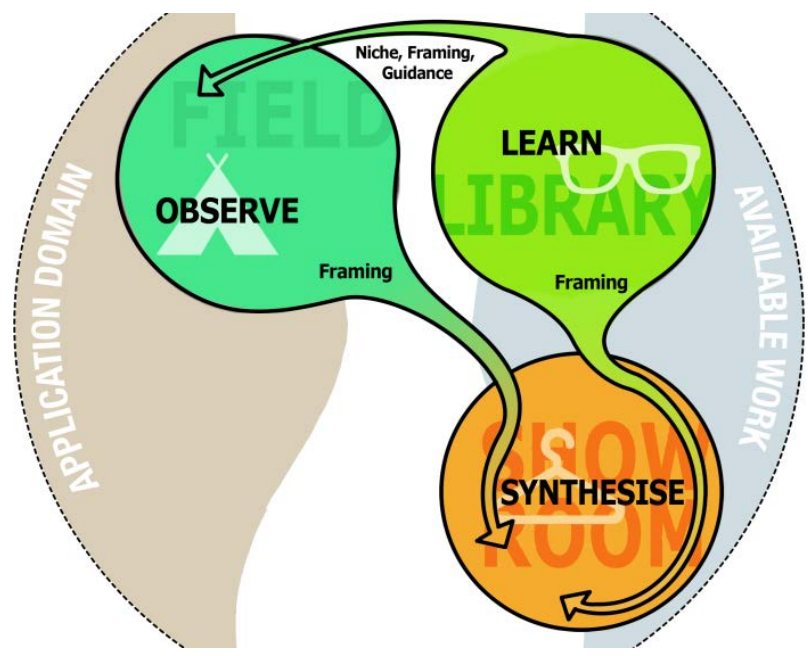

Figure 5: Triangulation path for field reframing papers.

\section{Special Cases and Combinations}

An inspiration oriented lab study can act as a replacement to the field study in this pattern to explore future rather than existing use-contexts i.e. [c]. In several papers the pattern was followed up by either a validated solution pattern (which we consider best practice - as it proves the utility of the novel insights), a workshop study (which illustrates the utility of the novel insights) or a lab study (which tests the novel insights against the application domain).

\section{Paradigm Papers}

A good example of the post-study literature review is found in the paper by Brown et al. [a] on implicit alignment work.
The inspiration lab approach is convincingly applied in [c] who studied remote assistance configurations. Their work also features a solid showroom study. The combination of a field reframing study with a validated solution approach is [d] in their work on hybrid augmented reality experiences .

\section{THREE CANDIDATE PATTERNS}

In this section we discuss the patterns for which we did not have enough papers to base a description on, but for which we were confident enough to assume a pattern could be formed - given enough data.

\section{Parameter Discovery}

A small batch of lab-centric papers investigated specific hypotheses or uncovered parameters which were useful groundwork for systems development or theory testing. These papers used library and lab as most important research strategies, sometimes combined with lightweight field or workshop studies. The papers in this pattern did not deliver novel theory (such as in the field-reframing pattern) and no new applications. All could be described as closed design canvas [10] papers.

\section{Transformative Design}

Creswell \& Clark [12] refer to transformative designs as studies that have an emancipatory agenda on top of the scientific agenda. Studies like this are found HCI, in particular in 'the Scandinavian school of participatory design' [e.g. 6]. We found only three papers in which such a social agenda was the most important contribution of the paper. These papers were hard to classify. They presented interventions such as introducing a design activity or system, in a context, which served multiple goals. From an HCI point of view they aimed at trying or testing the proposed method or solution. From a social point of view (which was clearly important to the authors) the same methods could be seen differently.

\section{Relevance Cycle}

Considering the status of user-centered design in our community it was somewhat surprising to find only one paper in our set featuring a full relevance cycle: fieldworkshop-lab. This may show an academic bias, which is not present in industry: solutions following a full relevance cycle are considered of less interest to academia from the perspective of the growth of knowledge.

\section{CONCLUSIONS \& DISCUSSION}

Within this paper we have shown that much of the research as it is done in HCI can be described as mixed-method research within the DOT-framework. We have found indications for how different research aims, and corresponding challenges, are supported by specific mixedmethod research designs. The patterns consolidate the pragmatic research practices of HCI researchers. Moreover, apart from the typology of research strategies as it is proposed by the DOT-framework, we have developed terminology to describe how the individual methods of a mixed-method setup can fit together - thus supporting discourse about the coherence of a research approach. 
Although, unsurprisingly, the dominant patterns as we found them appear to correspond to the traditions which are typically reported to form the makeup of HCI: science (field reframing, parameter discovery), engineering (validated solution) and design (rigor cycle), the DOT-framework places the approaches of these different traditions under the umbrella of a single theory, which may improve their combinability. Indeed, apart from describing how the different research strategies within a pattern can be coherently combined, we were able to suggest how patterns as a whole could be optimized for a follow up with a different pattern. This brings clarity about the intersections of different approaches, on a concrete methodological level, and it allows for well-founded debate on the pragmatics of cross-disciplinary research approaches.

Overall, the DOT-framework fitted the actual work, as it was reported in our sample of the NordiCHI 2012 proceedings well. All five research strategies were found frequently and most papers combined approaches directed at rigor and relevance, overview and certainty, and inspiration and data; triangulation appears to be the norm in HCI research, not the exception. From our data it seems that library and showroom studies are typically not regarded as 'studies' by HCI researchers. The sections containing related work or new theory and guidelines - additions to the domain of existing work, were often 'results only' sections. As these sections form an important part of the make-up of the paper, we feel our community has much to gain from a more methodological execution and reporting of library and -in particular- showroom studies.

We were initially surprised to find only one paper with a full relevance cycle (field, workshop, lab) in our dataset. Considering the continued dedication of HCI textbooks to the user-centered design cycle [11], which corresponds closely to the relevance cycle, one might expect successful examples of such an approach to be present in our conferences. There can be multiple reasons for the omission. One hypothesis is that, in a future oriented discipline, case- studies turn out to be an inefficient way to progress the state of the art, while it is still vital to the practice of HCI outside the scientific research community. This would have to be tested, for example by applying the DOT-framework to HCI work such as it executed in the industry practice of web, game and application design. Such a study about research pragmatics may uncover other biases in our current study such as the possible linearization of highly iterative research practices [27] through the processes of scientific storytelling.

Much in the way contemporary work on mixed-method research in the social sciences strikes a middle ground between the axiological debates of the paradigm wars and a vague 'anything goes' form of pragmatism (as Bergman [3] puts it). Our work provides a third route between those extremes. Although the patterns as we describe them here need to evolve further and will have to withstand the test of thoughtful application by researchers, in this paper we have already shown the outlines of a theory based, practice informed approach to research pragmatics in our multidisciplinary field.

\section{REFERED PAPERS FROM THE DATASET}

These papers appeared in Proceedings of the 7th Nordic Conference on Human-Computer Interaction: Making Sense Through Design (NordiCHI '12). ACM, New York, NY, USA.

a. Brown, J. M., Lindgaard, G. and Biddle, R. Joint implicit alignment work of interaction designers and software developers. 693-702

b. Dalsgaard, P. and Halskov, K. Tangible 3D tabletops: combining tangible tabletop interaction and 3D projection. 109-118.

c. Giusti, L., Xerxes, K., Schladow, A., Wallen, N., Zane, F. and Casalegno, F. Workspace configurations: setting the stage for remote collaboration on physical tasks. 351-360.

d. Grubert, J., Grasset, R. and Reitmayr, G. Exploring the design of hybrid interfaces for augmented posters in public spaces. 238-246.

e. Gonzalez, B., Carroll, E. and Latulipe, C. Danceinspired technology, technology-inspired dance. 398407.

f. Hansen, J. P., Glenstrup, A. J., Wusheng, W., Weiping, L. and Zhonghai, W. Collecting location-based voice messages on a TalkingBadge. 219-227.

g. Laporte, L., Slegers, K. and De Grooff, D. Using Correspondence Analysis to monitor the persona segmentation process. 265-274.

\section{OTHER REFERENCES}

1. Alexander, C., Ishikawa, S. and Silverstein, M. $A$ Pattern Language: Towns, Buildings, Construction. Center for Environmental Structure, 1977

2. Bartneck, C. and Rauterberg, M. HCI reality: an "Unreal Tournament"'TM? International Journal of HumanComputer Studies, 65, 8 ( 2007), 737-743.

3. Bergman, M. M. Advances in mixed methods research: Theories and applications. Sage, 2008.

4. Bødker, S. When second wave HCI meets third wave challenges. In Proceedings of NordiCHI 2006, 1-8.

5. Borchers, J. O. A pattern approach to interaction design. In AI \& Society, 15, 4 (2001), 359-376.

6. Blomberg, J. and Karasti, H. Positioning ethnography within Participatory Design. In Routledge Handbook of Participatory Design, 2012, 86-116.

7. Bryman, A. Why do researchers integrate/combine/ $\mathrm{mesh} / \mathrm{blend} / \mathrm{mix} / \mathrm{merge} /$ fuse quantitative and qualitative research. Advances in mixed methods research, (2008), 87-100. 
8. Carroll, J. M. and Kellogg, W. A. Artifact as theorynexus: Hermeneutics meets theory-based design. In Proceedings of CHI 1989, 7-14.

9. CHI 2013 Contribution Types. Available from: http://chi2013.acm.org/authors/call-forparticipation/papers-notes/selecting-a-contribution-type/

10. Cockton, G. Make Evaluation Poverty History. In Proceedings of CHI 2007 (Alt.Chi), ACM

11. Cockton, G. Revisiting usability's three key principles. In Proceedings of CHI 2008, ACM, 2473-2484

12. Creswell, J. W. and Clark, V. L. P. Designing and conducting mixed methods research. Wiley Online Library, 2007.

13. Dorst, C. H. Describing Design-A comparison of paradigms. $\mathrm{PhD}$ Thesis Delft University of Technology (1997).

14. Fallman, D. The interaction design research triangle of design practice, design studies, and design exploration. Design Issues, 24, 3 ( 2008), 4-18.

15. Gaver, W. What should we expect from research through design? In Proceedings of CHI 2012. ACM, 937-946.

16. Greene, J. C., Caracelli, V. J. and Graham, W. F. Toward a conceptual framework for mixed-method evaluation designs. Educational evaluation and policy analysis, 11, 3 (1989), 255-274.

17. Gregor, S. and Hevner, A. R. Positioning and Presenting Design Science Research for Maximum Impact. MIS Quarterly, 37, 2 (2013).

18. Grudin, J. Three faces of human-computer interaction. Annals of the History of Computing, IEEE, 27, 4 (2005), 46-62.

19. Gwet, K. Handbook of inter-rater reliability. Gaithersburg, MD: STATAXIS Publishing Company, (2001), 223-246.

20. Harrison, S., Tatar, D. and Sengers, P. The three paradigms of HCI. In Proceedings of CHI 2007 ACM. pp

21.Helm, R., Johnson, R., Vlissides, J. and Gamma, E. Design Patterns: Elements of Reusable Object-Oriented Software. Addison-Wesley, 2002.

22. Hevner, A. R., March, S. T., Park, J. and Ram, S. Design science in information systems research. MIS quarterly, 28, 1 (2004), 75-105.

23. Järvinen, P. Research Questions Guiding Selection of an Appropriate Research Method. In ECIS (2000), 124131.

24. Jarvis, N., Cameron, D. and Boucher, A. Attention to detail: annotations of a design process. In proceedings of NordiCHI 2012, ACM, 11-20.
25. Koskinen, I., Zimmerman, J., Binder, T., Redstrom, J. and Wensveen, S. Design research through practice: From the lab, field, and showroom. Elsevier, 2011.

26. Mackay, W. E. and Fayard, A. HCI, natural science and design: a framework for triangulation across disciplines. Proceedings of DIS 1997. ACM, 223-234.

27. McKenney, S. and Reeves, T. Conducting educational design research. (2011).

28. Mingers, J. Combining IS research methods: towards a pluralist methodology. Information systems research, 12, 3 (2001), 240-259.

29. Newman, W. A preliminary analysis of the products of HCI research, using pro forma abstracts. Proceedings of CHI 1994. ACM, 278-284.

30. Nunamaker Jr, J. F. and Chen, M. Systems development in information systems research. In Proceedings of System Sciences. IEEE, 1990, 631-640.

31. Oates, B. J. Researching information systems and computing. Sage, 2005.

32. Pan, Y. and Stolterman, E. Pattern language and HCI: expectations and experiences. In Proceedings of CHI 2013, ACM, 1989-1998.

33. Petter, S. C. and Gallivan, M. J. Toward a framework for classifying and guiding mixed method research in information systems. Proceedings of System Sciences 2004. IEEE

34. Rauterberg, M. HCI as an engineering discipline: to be or not to be!? African Journal of Information \& Communication Technology, 2, 4 ( 2006), 22.

35. Runkel, P. J. and MacGrath, J. E. Research on human behavior. Holt, Rinehart and Winston. New York, 1972

36. Shaw, M. What makes good research in software engineering? International Journal on Software Tools for Technology Transfer, 4, 1 ( 2002), 1-7.

37. Turnhout, K. van, Craenmehr, S. Holwerda, R., Menijn, M., Zwart, J.P. and Bakker, R. Trade-offs in Design Research: Development Oriented Triangulation. Proceedings of the BSC HCI Conference 2013.

38. Whittaker, S., Terveen, L. and Nardi, B. A. Let's stop pushing the envelope and start addressing it: a reference task agenda for HCI. Human-Computer Interaction, 15, 2-3 (2000), 75-106.

39. Wolf, T. V., Rode, J. A., Sussman, J. and Kellogg, W. A. Dispelling design as the black art of CHI. In Proceedings of CHI 2006. ACM, 521-530.

40.Zimmerman, J., Forlizzi, J. and Evenson, S. Research through design as a method for interaction design research in HCI. In Proceedings of CHI 2007. ACM, 493-502. 